International Mathematical Forum, Vol. 8, 2013, no. 18, 865 - 873

HIKARI Ltd, www.m-hikari.com

\title{
Asymptotic Frequencies of Beams Left Fixed and Right Supported
}

\author{
A. M. Marin \\ Faculty of Exact and Natural Sciences, University of Cartagena \\ Sede Piedra de Bolivar, Avenue of Consulado \\ Cartagena de Indias, Bolivar, Colombia \\ R. D. Ortiz \\ Faculty of Exact and Natural Sciences, University of Cartagena \\ Sede Piedra de Bolivar, Avenue of Consulado \\ Cartagena de Indias, Bolivar, Colombia
}

\section{J. A. Rodriguez}

Morelia Institute of Technology, Morelia, Mexico

Copyright (c) 2013 A. M. Marin et al. This is an open access article distributed under the Creative Commons Attribution License, which permits unrestricted use, distribution, and reproduction in any medium, provided the original work is properly cited.

\begin{abstract}
The purpose of this paper is to construct the asymptotic for natural frequencies of the Euler-Bernoulli beam problem with non-uniform section using the method of Wentzel-Kramers-Brillouin (WKB) and find the secular equation for the eigenvalues a non-uniform beam embedded at one end and supported at the other end.
\end{abstract}

Mathematics Subject Classification: 35B40

Keywords: WKB method; Euller-Bernoulli beam 


\section{Introduction}

The beam is one of the structural elements most used. Its importance lies in the strength of materials, the completeness of which is taught in schools stress analysis techniques in the beams, so as its wide use in all types of bridges, buildings, trucks, etc.

Therefore, the vibration of beams has been studied extensively and continues to receive attention in the current literature. Initially, Daniel Bernoulli in the eighteenth century became interested for determining the elasticity of the rods, the vibrations of the beams and developing the differential equation for the vibration a bar. Leonhard Euler was born in the eighteenth century in Basel (Switzerland), influenced by Daniel Bernoulli study problems elasticity of the beams and columns, use the method of minimum work and contributed his valuable discussion on "buckling of the columns."

(Mabie, Rogers [6], 1974) studied various cases of vibrations cantilevered transverse beams with different conditions ends. (Naguleswaran [7], 1994) found a solution for transverse vibration of Euler Bernoulli beams and conical beams. In (Abrate [1], 1995), were obtained natural frequencies Euler Bernoulli beams (EB) when solution of the equation of EB was expressed by elementary functions. In (Hsu, Lai, Chen [5], 2008) was studied vibration of non-uniform beams using the Euler Bernoulli modified decomposition method of Adomian.

For the equation of the beam with a uniform small parameter, you can use the method (WKB) Wentzel - Kramers - Brillouin, also known in the literature as the approximation Liouville - Green. In (Akulenko, Nesterov [2], 2005) WKB method was used for finding asymptotic high frequency, this method is to obtain asymptotic series for solutions powers with a small parameter.

Although the WKB method is developed for differential equations of $n$ th order (Fedoryuk [4], 1993), this technique was applied in the equation EB fourth order in (Aya, Cano, Zhevandrov [3], 2011) to non-uniform embedded and cantilevered beams.

In the next section one takes the Bernoulli Euler equation, for non-uniform beams.

\section{Mathematical formulation}

In this section we find the approximate solution as a linear combination of four linearly independent solutions. After imposing the boundary conditions for the case of fixed at one end and supported at the other, which is reduced to a system of four equations with four unknowns which has nontrivial solutions if its determinant is zero. And finally we obtain the asymptotic determinant equation when $\epsilon \rightarrow 0$. Then we have eigenvalues. 


\section{$2.1 \quad$ Euler-Bernoulli equation}

We take the Euler-Bernoulli equation for transverse vibrations in the nonuniform beam approximation have been described by (Tijonov-Samarsky [8], 1972)

$$
\rho S(x) \frac{\partial^{2} y}{\partial t^{2}}+\frac{\partial^{2}}{\partial x^{2}}\left(E J(x) \frac{\partial^{2} y}{\partial x^{2}}\right)=0,
$$

where $E$ is the Young's modulus of the beam, $\rho$ is the density, $S(x)$ is the cross-sectional area, $J(x)$ is the moment of inertia of the cross section with respect to its horizontal axis and $y$ is the transverse displacement. It is assumed that functions $S(x)$ and $J(x)$ are smooth and positive $0 \leq x \leq l$ where $l$ is the length of the beam.

Looking for the solution of the equation of Bernoulli (1) in the form

$$
y(x, t)=e^{i \omega t} v(x),
$$

where $w$ is the frequency. Substituting (2) in the equation (1) we obtain fourth order ordinary differential equation for $v(x)$

$$
-\omega^{2} \rho S(x) v(x)+\left(E J(x) v^{\prime \prime}(x)\right)^{\prime \prime}=0 .
$$

Taking the boundary conditions for beams fixed in an end and supported at the other. Equation (3) is transformed into the following problem:

$$
\begin{aligned}
& \frac{1}{\rho S(x)}\left(E J(x) v^{\prime \prime}(x)\right)^{\prime \prime}=\omega^{2} v(x) . \\
& v(0)=v(l)=0, v^{\prime}(0)=v^{\prime \prime}(l)=0 .
\end{aligned}
$$

The discrete spectrum of the problem (EB) (4) constitutes a sequence $w_{n}$ of real numbers tending to infinity when $n \rightarrow \infty$. In view of the above can be consider $w^{2}=\frac{1}{\epsilon^{4}}$, where $\epsilon \rightarrow 0$ (Akulenko, Nesterov [2], 2005). Therefore the problem (EB), (4) becomes

$$
\begin{aligned}
& \frac{1}{\rho S(x)}\left(E J(x) v^{\prime \prime}(x)\right)^{\prime \prime}=\frac{1}{\epsilon^{4}} v(x) . \\
& v(0)=v(l)=0, v^{\prime}(0)=v^{\prime \prime}(l)=0 .
\end{aligned}
$$

In the next section we calculate the secular equation of the problem (5)-(6). 


\section{Main result}

This section states and solves the problem of Euler Bernoulli beams at high frequency, which is applied the WKB method.

The mathematical formulation of the problem is the searching for nontrivial solutions of the problem (5)-(6).

The main result is as follows

Theorem 3.1. The eigenvalues of the problem (5)-(6) are given by $w_{n}^{2}=$ $\frac{1}{\varepsilon_{n}^{4}}$, with

$$
\omega_{n}=\left(\frac{(4 n+1) \pi}{4 \int_{0}^{l} a^{-\frac{1}{4}}(x) d x}\right)^{2}\left(1+O\left(\frac{1}{n}\right)\right), \quad n=0,1, \ldots, \quad n \rightarrow \infty,
$$

where $\varepsilon_{n}$ is the solution of the secular equation (25). The function $a^{-\frac{1}{4}}(x)$ is defined by equation (23)

Proof. Given that $\omega^{2}=\frac{1}{\varepsilon^{4}}$ then equation (5) can be transformed into

$$
\begin{gathered}
v(x)=\frac{\varepsilon^{4}}{\rho S(x)}\left(E J(x) v^{\prime \prime}(x)\right)^{\prime \prime} \\
v(x)=\frac{\varepsilon^{4}}{\rho S(x)}\left(E J^{\prime \prime}(x) v^{\prime \prime}(x)+2 E J^{\prime}(x) v^{\prime \prime \prime}(x)+E J(x) v^{\prime \prime \prime \prime}(x)\right) .
\end{gathered}
$$

Following the traditional WKB method, the analytical solution approximates equation (5) can be replaced by a power series given by the following

$$
v(x)=A(x, \varepsilon) e^{\frac{i \phi(x)}{\varepsilon}}, \quad \varepsilon \rightarrow 0,
$$

where

$$
A(x, \varepsilon)=A_{0}(x)+\varepsilon A_{1}(x)+\varepsilon^{2} A_{2}(x)+\ldots, \quad \varepsilon \rightarrow 0,
$$

with $\phi(x)$ and $A_{j}(x), j=0,1,2, \ldots$ are smooth functions and unknown.

Replacing (8) and each of the derivatives of $v(x)$ in $(7)$, we have the following expression

$$
\begin{aligned}
A(x, \varepsilon)= & \frac{E}{\rho S(x)}\left[J(x) \phi_{x}^{4}(x) A(x, \varepsilon)-2 i \varepsilon\left(2 J(x) \phi_{x}^{3}(x) A_{x}(x, \varepsilon)\right.\right. \\
& \left.\left.+\left(J_{x}(x) \phi_{x}^{3}(x)+J(x) 3 \phi_{x}^{2}(x) \phi_{x x}(x)\right) A(x, \varepsilon)\right)\right]+O\left(\varepsilon^{2}\right) \\
= & \frac{E}{\rho S(x)}\left[J(x) \phi_{x}^{4}(x) A(x, \varepsilon)-2 i \varepsilon\left(2 J(x) \phi_{x}^{3}(x) A_{x}(x, \varepsilon)\right.\right. \\
& \left.\left.+\left(J(x) \phi_{x}^{3}(x)\right)^{\prime} A(x, \varepsilon)\right)\right]+O\left(\varepsilon^{2}\right), \quad \varepsilon \rightarrow 0 .
\end{aligned}
$$


Replacing (9) on both sides of (10) we obtain

$$
\begin{aligned}
A_{0}(x)+\varepsilon A_{1}(x) & +\varepsilon^{2} A_{2}(x)+\cdots=\frac{E}{\rho S(x)}\left[J ( x ) \phi _ { x } ^ { 4 } ( x ) \left(A_{0}(x)+\varepsilon A_{1}(x)\right.\right. \\
\left.+\varepsilon^{2} A_{2}(x)+\cdots\right)- & 2 i \varepsilon\left(2 J(x) \phi_{x}^{3}(x)\left(A_{0 x}(x)+\varepsilon A_{1 x}(x)+\varepsilon^{2} A_{2 x}(x)+\cdots\right)\right) \\
& \left.+\left(J(x) \phi_{x}^{3}(x)\right)^{\prime}\left(A_{0}(x)+\varepsilon A_{1}(x)+\varepsilon^{2} A_{2}(x)+\cdots\right)\right]+O\left(\varepsilon^{2}\right) \\
= & \frac{E J(x)}{\rho S(x)} \phi_{x}^{4}(x) A_{0}(x)+\varepsilon\left[\frac{E J(x)}{\rho S(x)} \phi_{x}^{4}(x) A_{1}(x)\right. \\
& \left.-\frac{4 i E J(x)}{\rho S(x)} \phi_{x}^{3}(x) A_{0 x}(x)-\frac{2 i E}{\rho S(x)}\left(J(x) \phi_{x}^{3}(x)\right)^{\prime} A_{0}(x)\right] \\
& +O\left(\varepsilon^{2}\right), \quad \varepsilon \rightarrow 0 .
\end{aligned}
$$

Equating the coefficients of the asymptotic series in $\varepsilon$ and taking corresponding to $\varepsilon^{0}$ in (12) and using that $A_{0} \neq 0$ as seen in the equation (16), it can be obtained

$$
\varepsilon^{0}: \frac{E J(x)}{\rho S(x)} \phi_{x}^{4}(x)=1 .
$$

From equation (13) and choosing the corresponding equality to $\varepsilon^{1}$ in (12), we obtain

$$
\varepsilon^{1}: 2 J(x) \phi_{x}^{3}(x) A_{0 x}(x)+\left(J(x) \phi_{x}^{3}(x)\right)^{\prime} A_{0}(x)=0 .
$$

Equating the asymptotic series are obtained more equations but we will consider only the first two equations, other equations are of order $O\left(\varepsilon^{2}\right)$. Since equation (13) has two real roots and two pure imaginary roots with opposite signs, we obtain

$$
\phi_{k}(x)=(i)^{k} \int\left(\frac{E J(x)}{\rho S(x)}\right)^{-\frac{1}{4}} d x, \quad k=1,2,3,4 .
$$

From the equation (14) and separating the functions $A_{0}(x), J(x) \phi_{x}(x)^{3}$ and integrating on both sides, it follows that

$$
A_{0}(x)=C\left(J(x) \phi_{x}(x)^{3}\right)^{-\frac{1}{2}}
$$


where $C$ is a non-zero arbitrary constant. it Therefore, differentiating with respect to $x$ in the equation (15) and substituting (16), function $A_{0}(x)$ can be expressed as follows

$$
\begin{aligned}
A_{0}(x) & =C\left(J(x)\left(\frac{J(x)}{S(x)}\right)^{-\frac{3}{4}}\right)^{-\frac{1}{2}} \\
& =C\left(J^{\frac{1}{4}}(x) S^{\frac{3}{4}}(x)\right)^{-\frac{1}{2}} \\
& =C J^{-\frac{1}{8}}(x) S^{-\frac{3}{8}}(x) .
\end{aligned}
$$

Therefore, replacing (15) and (17) in (8) which is the solution $v(x)$ of (5), we have

$$
\begin{gathered}
v_{1}(x)=d_{1} J^{-\frac{1}{8}}(x) S^{-\frac{3}{8}}(x) \sin \left(\frac{\psi_{1}(x)}{\varepsilon}\right)+O(\varepsilon), \quad \varepsilon \rightarrow 0, \\
v_{2}(x)=d_{2} J^{-\frac{1}{8}}(x) S^{-\frac{3}{8}}(x) \cos \left(\frac{\psi_{1}(x)}{\varepsilon}\right)+O(\varepsilon), \quad \varepsilon \rightarrow 0, \\
v_{3}(x)=d_{3} J^{-\frac{1}{8}}(x) S^{-\frac{3}{8}}(x) e^{-\frac{\psi_{1}(x)}{\varepsilon}}+O(\varepsilon), \quad \varepsilon \rightarrow 0, \\
v_{4}(x)=d_{4} J^{-\frac{1}{8}}(x) S^{-\frac{3}{8}}(x) e^{-\frac{\psi_{2}(x)}{\varepsilon}}+O(\varepsilon), \quad \varepsilon \rightarrow 0,
\end{gathered}
$$

and writing the solution (5) as the first term of the linear combination of $v_{1}(x), v_{2}(x), v_{3}(x), v_{4}(x)$

$$
\begin{aligned}
& v(x)=J^{-\frac{1}{8}}(x) S^{-\frac{3}{8}}(x) \\
& \left(c_{1} \sin \left(\frac{\psi_{1}(x)}{\varepsilon}\right)+c_{2} \cos \left(\frac{\psi_{1}(x)}{\varepsilon}\right)+c_{3} e^{-\frac{\psi_{1}(x)}{\varepsilon}}+c_{4} e^{-\frac{\psi_{2}(x)}{\varepsilon}}\right),
\end{aligned}
$$

$\varepsilon \rightarrow 0$, where

$$
\psi_{1}(x)=\int_{0}^{x} a^{-\frac{1}{4}}(t) d t, \quad \psi_{2}(x)=\int_{x}^{l} a^{-\frac{1}{4}}(t) d t, \quad a(t)=\frac{E J(t)}{\rho S(t)},
$$

and $c_{1}, c_{2}, c_{3}, c_{4}$ are constants. It is noted that $\sin \left(\frac{\psi_{1}}{\varepsilon}\right), \cos \left(\frac{\psi_{1}}{\varepsilon}\right), e^{-\frac{\psi_{1}}{\varepsilon}}, e^{-\frac{\psi_{2}}{\varepsilon}}$ are linearly independent of the fact that $\psi_{2}(x)=L-\psi_{1}(x)$, where $L$ is defined in $(26)$ and since $\sin \left(\frac{\psi_{1}}{\varepsilon}\right), \cos \left(\frac{\psi_{1}}{\varepsilon}\right), e^{-\frac{\psi_{1}}{\varepsilon}}, e^{\frac{\psi_{1}}{\varepsilon}}$ are linearly independent. 
The boundary conditions for a beam fixed on the left and supported on the right side are given by

$$
v(0)=v(l)=0, \quad \varepsilon v^{\prime}(0)=\varepsilon^{2} v^{\prime \prime}(l)=0 .
$$

From the solution (22) and boundary conditions (24), yields a homogeneous system of four equations for four constants $c_{i}, i=1,2,3,4$. This system has nontrivial solutions when

$$
\left|\begin{array}{cccc}
0 & -1 & -1 & -e^{-\frac{L}{\varepsilon}} \\
-\operatorname{sen}\left(\frac{L}{\varepsilon}\right) & -\cos \left(\frac{L}{\varepsilon}\right) & -e^{-\frac{L}{\varepsilon}} & -1 \\
-\gamma & \frac{\alpha \varepsilon}{8} & \gamma+\frac{\alpha \varepsilon}{8} & \left(\frac{\alpha \varepsilon}{8}-\gamma\right) e^{-\frac{L}{\varepsilon}} \\
R_{1} & R_{2} & R_{3} & R_{4}
\end{array}\right|=0
$$

where

$$
\begin{aligned}
& L=\int_{0}^{l} a^{-\frac{1}{4}}(t) d t, \quad \alpha=J^{-1}(0) J^{\prime}(0)+3 S^{-1}(0) S^{\prime}(0), \quad \theta=\left(\psi_{1}^{\prime}(l)\right)^{2}, \\
& \beta=\frac{3\left(S^{\prime \prime}(l)\right)}{8 S(l)}-\frac{33\left(S^{\prime}(l)\right)^{2}}{64 S(l)^{2}}-\frac{3 J^{\prime}(l) S^{\prime}(l)}{32 J(l) S(l)}+\frac{J^{\prime \prime}(l)}{8 J(l)}-\frac{9\left(J^{\prime}(l)\right)^{2}}{64 J(l)^{2}} \\
& \mu=-\psi_{1}^{\prime \prime}(l)+\frac{\psi_{1}^{\prime}(l) J^{\prime}(l)}{4 J(l)}+\frac{3 \psi_{1}^{\prime}(l) S^{\prime}(l)}{4 S(l)}, \quad \gamma=a^{-\frac{1}{4}(0),}
\end{aligned}
$$

and

$$
\begin{aligned}
& R_{1}=\left(\theta+\beta \varepsilon^{2}\right) \operatorname{sen}\left(\frac{L}{\varepsilon}\right)+\mu \varepsilon \cos \left(\frac{L}{\varepsilon}\right) \\
& R_{2}=-\mu \varepsilon \operatorname{sen}\left(\frac{L}{\varepsilon}\right)+\left(\theta+\beta \varepsilon^{2}\right) \cos \left(\frac{L}{\varepsilon}\right) \\
& R_{3}=\left(-\theta-\mu \varepsilon+\beta \varepsilon^{2}\right) e^{-\frac{L}{\varepsilon}} \\
& R_{4}=-\theta+\mu \varepsilon+\beta \varepsilon^{2}
\end{aligned}
$$

The equation (25) is the secular equation for natural frequency $\omega_{n}=\varepsilon_{n}^{-2}$.

They get the asymptotic fundamental system of solutions with the precision $O(\varepsilon)$, when $\varepsilon \rightarrow 0$, we have to eliminate higher order terms (e.g, $e^{-\frac{L}{\varepsilon}}=$ $\left.O\left(\varepsilon^{\infty}\right), \quad \varepsilon \rightarrow 0\right)$ en (25). It is understood that $O\left(\varepsilon^{\infty}\right)$ is the same as $O\left(\varepsilon^{n}\right)$ for all $n \in \mathbb{N}$. Therefore the secular equation (25) is

$$
\left|\begin{array}{cccc}
0 & 1 & 1 & O\left(\varepsilon^{\infty}\right) \\
\operatorname{sen}\left(\frac{L}{\varepsilon}\right) & \cos \left(\frac{L}{\varepsilon}\right) & O\left(\varepsilon^{\infty}\right) & 1 \\
1 & O(\varepsilon) & -1+O(\varepsilon) & O\left(\varepsilon^{\infty}\right) \\
-\operatorname{sen}\left(\frac{L}{\varepsilon}\right)+O(\varepsilon) & -\cos \left(\frac{L}{\varepsilon}\right)+O(\varepsilon) & O\left(\varepsilon^{\infty}\right) & 1+O(\varepsilon)
\end{array}\right|=0 \text {. }
$$


Therefore

$$
\tan \left(\frac{L}{\varepsilon}\right)=1+O(\varepsilon), \quad \varepsilon \rightarrow 0, \quad \varepsilon=\varepsilon_{n}=\frac{4 L}{(4 n+1) \pi}(1+O(\varepsilon))
$$

then

$$
\omega_{n}=\left(\frac{(4 n+1) \pi}{4 \int_{0}^{l} a^{-\frac{1}{4}}(t) d t}\right)^{2}\left(1+O\left(\frac{1}{n}\right)\right), \quad n \rightarrow \infty, n=0,1, \ldots
$$

From (27) and initial conditions, the eigenfunction is

$$
v_{n}(x)=c_{3} J^{-\frac{1}{8}}(x) S^{-\frac{3}{8}}(x)\left(\operatorname{sen}\left(\frac{\psi_{1}(x)}{\varepsilon_{n}}\right)-\cos \left(\frac{\psi_{1}(x)}{\varepsilon_{n}}\right)+e^{-\frac{\psi_{1}(x)}{\varepsilon_{n}}}\right),
$$

where $c_{3}$ is an arbitrary constant.

ACKNOWLEDGEMENTS. The authors express their deep gratitude to CONACYT-México, Programa de Mejoramiento del Profesorado (PROMEP)México and Universidad de Cartagena for financial support.

\section{References}

[1] S. Abrate, Vibration of non-uniform rods and beams, Journal of Sound and Vibration, 185 (1995), 703-716.

[2] L. P. Akulenko and S. V. Nesterov, High-precision Methods in Eingenvalue Problems and Their Applications, Chapman Hall, 2005.

[3] H. Aya, R. Cano and P. Zhevandrov, Frecuencias propias de vigas de Euler-Bernoulli no uniformes, Ingeniería e Investigación, Universidad Nacional de Colombia, 2011.

[4] M. V. Fedoryuk, Asymptotic Analysis. Linear Ordinary Differential Equations, Springer, 1993.

[5] J. Ch. Hsu, H. Y. Lai, and C. K. Chen, Free vibration of non-uniform Euler-Bernoulli beams with general elastically end constraints using Adomian modified decomposition method, Journal of Sound and Vibration, 318 (2008), 965-981.

[6] H. H. Mabie and C. B. Rogers, Transverse vibrations of double-tapered cantilever beams with end support and with end mass, Journal of the acoustical society of America, 552 (1974), 986-991. 
[7] S. Naguleswaran, A direct solution for the transverse vibration of EulerBernoulli wedge and cone beams, Journal of Sound and Vibration, 1723 (1994), 289-304.

[8] A. N. Tijonov and A. A. Samarsky, Ecuaciones de la Física Matemática, Editorial Mir-Moscu, 1972.

Received: March 25, 2013 